\title{
Temperature dependence in random matrix models with pairing condensates
}

\author{
Benoît Vanderheyden \\ Department of Electrical Engineering and Computer Science, \\ Montefiore Bât. B-28, Université de Liège, \\ B-4000 Liège (Sart-Tilman), Belgium \\ A. D. Jackson \\ The Niels Bohr Institute, Blegdamsvej 17, DK-2100 Copenhagen Ø, Denmark
}

(Dated: November 17, 2018)

\begin{abstract}
We address a number of issues raised by a manuscript of Klein, Toublan, and Verbaarschot (hep-ph/0405180) in which the authors introduce a random matrix model for QCD with two colors, two flavors, and fermions in the fundamental representation. Their inclusion of temperature terms differs from the approach adopted in previous work on this problem (Phys. Rev. D 64, 074016 (2001).) We demonstrate that the two approaches are related by a transformation that leaves the thermodynamic potential invariant and which therefore has no effect on physical observables.
\end{abstract}

PACS numbers: 11.30. Fs, 11.30. Qc, 11.30. Rd, 12.38. Aw

A number of model calculations of QCD at finite temperature and non-zero baryon density have revealed the existence of a rich phase structure 1, 2, 3, 4]. A particularly interesting possibility arises when cold nuclear matter is brought to densities sufficiently large for quark degrees of freedom to become important. It is thought that, due to quark Cooper pairing, cold and dense quark matter behaves like a color superconductor [1, 2]. The system is characterized by a variety of color superconducting order parameters which have been studied as a function of, e.g., the total number of flavors, the quark masses, the quark chemical potentials, and temperature [3]. These results have not been confirmed satisfactorily by lattice simulations. Such calculations are difficult to perform and interpret at finite baryon densities. The fermion determinant appearing in the partition function is complex for non-zero baryon chemical potential, and standard Monte Carlo methods can no longer be applied. As a consequence, our theoretical understanding of the QCD phase diagram at non-zero baryon density remains incomplete.

Random matrix theory can provide interesting elementary models that capture important aspects of the thermodynamic competition among different order parameters and produce robust results. Such models respect the global symmetries of QCD but mimic the detailed dynamics of the interactions with a form that can be treated analytically. When supplemented by additional physical insight regarding the form and relative importance of order parameters, random matrix theory can efficiently model the physics of systems with non-trivial phase diagrams. In its usual implementation, the fermion kinetic energy is ignored; only the interactions and their symmetries are retained. Such models are thus intrinsically theories of low-energy degrees of freedom which are treated at the mean field level. Near critical regions, random matrix results are similar to those obtained in a Landau-Ginzburg approach [4, 5].

The effects of baryon density and temperature must be included in a manner that respects the structure of QCD. In the finite temperature formalism, the quark chemical potentials, $\mu_{f}$, and the temperature, $T$, enter the QCD partition function via the finite Matsubara frequencies $\mu_{f}+\omega_{n}=\mu_{f}+i(2 n+1) \pi T$ (with $n=0, \pm 1, \pm 2, \ldots$ ). The inclusion of density effects through the addition of a chemical potential term, $\psi^{\dagger} \mu \gamma_{4} \psi$, in the Lagrangian is straightforward. In contrast to lattice calculations, the resulting random matrix partition function can be evaluated analytically. Its extremization leads to gap equations which are polynomial in the condensation fields, and which can thus be solved analytically or numerically.

Different schemes have been proposed in the literature for the inclusion of temperature effects. We note first that, since the theory describes low-lying energy excitations, much of the critical physics is captured even if the sum over fermion frequencies is restricted to the two lowest terms, $\omega_{n}= \pm i \pi T$. When this truncation is adopted in a chiral random matrix theory with $\mu_{f}=0$, for example, the resulting model shows a second-order phase transition independent of the number of flavors [ 6 ]. Taking into account all terms in the frequency sum does not change this result fundamentally. The sole effect is the introduction of a temperature mapping that is inhomogeneous (i.e., it depends on $T$ ) but monotonic [7, [8]. The critical temperature is thus modified, but neither the topology of the phase diagram nor the order of the transition are affected. In the following, we will only consider cases where the Matsubara sum is truncated to the two smallest terms.

In an early random matrix model of color superconductivity in QCD with two flavors 8, 9], we employed a partition function for which the pairing fields had opposite frequencies, $\omega_{n}$. This choice was dictated by the physical observation that the pairing condensate, $\left\langle\psi^{T}\left(i \sigma_{2}\right)_{\text {flavor }}\left(i \sigma_{2}\right)_{\text {color }}\left(i \sigma_{2}\right)_{\text {spin }} \psi\right\rangle$ (with $i \sigma_{2}$ the antisymmetric Pauli matrix), is independent of time. A Fourier decomposition selects opposite frequencies for the two flavor fields. This frequency selection is operative independent of whether one is modeling $S U(3)$-QCD or $S U(2)$-QCD. A seemingly different 
temperature dependence, based on the symmetries of the interactions, was recently introduced by Klein, Toublan, and Verbaarschot [10, 11]. The temperature scheme proposed there reflects the structure of the zero-temperature chiral random matrix models. For $S U(2)$ with fundamental fermions, which is characterized by real matrix elements, the temperature terms are chosen to be real. Similarly, both the temperature terms and the random matrix elements are complex for $S U(3)$ with fermions in the fundamental representation.

There would thus appear to be two different ways to treat temperature effects. The purpose of this paper is to demonstrate that the scheme based on the symmetries of the interactions is actually equivalent to that based on frequency selection. After summarizing the two temperature schemes in Sec. I we consider the transformation that relates them for the case of QCD with two colors in Sec. II The case of QCD with three colors is discussed in Sec. III

\section{TEMPERATURE SCHEMES}

Random matrix models that are capable of studying the competition between chiral, diquark, and other condensation channels are extensions of the chiral random matrix models $[4,6,12,13]$. In the sector of zero topological charge and for $N_{f}$ flavors of fermions with masses $m_{f}$, the partition function has the form

$$
Z_{0}=\prod_{f} \int \mathcal{D} \psi_{f}^{\dagger} \mathcal{D} \psi_{f} \mathcal{D} W e^{-\frac{n \beta \Sigma^{2}}{2}} \operatorname{Tr} W W^{\dagger} \exp \left(\sum_{f=1}^{N_{f}} \psi_{f}^{\dagger}\left(D[W]+m_{f}\right) \psi_{f}\right),
$$

where $W$ is an $n \times n$ matrix which models the interaction. The elements of $W$ are drawn on a Gaussian distribution of inverse variance $\Sigma$. Here, $n$ is a measure of the number of low energy degrees of freedom and is to be taken to infinity at the end of the calculations (i.e., in the thermodynamic limit). The Dirac operator, $D[W]$, respects the chiral symmetry of QCD, $\left\{D, \gamma_{5}\right\}=0$. In the basis of the eigenstates of $\gamma_{5}, D[W]$ can be decomposed as

$$
D[W]=\left(\begin{array}{cc}
0 & i W \\
i W^{\dagger} & 0
\end{array}\right)
$$

For random matrix models of QCD with $S U(3)$ and fermions in the fundamental representation, $W$ is complex. This choice corresponds to a chiral unitary ensemble characterized by a Dyson index $\beta=2$. The QCD Dirac operator in $S U(2)$ with fermions in the fundamental representation satisfies an additional anti-unitary symmetry,

$$
\left[C\left(\sigma_{2}\right)_{\text {color }} K, i D\right]=0,
$$

with $\left(C\left(\sigma_{2}\right)_{\text {color }} K\right)^{2}=1$. ( $C$ is the charge conjugation operator, $\sigma_{2}$ the antisymmetric color matrix, and $K$ the complex conjugation operator.) This leads to a particular basis of states in which $D$ is real. Accordingly, $W$ is chosen real in chiral random matrix models for $S U(2)$, and the model belongs to the chiral orthogonal ensemble with an index $\beta=1$ [14]. The essential difference between the unitary and orthogonal ensembles lies in the number of independent random variables that are allowed per matrix element. (I.e., A complex number has two degrees of freedom; a real number has only one.) This difference is important in determining the statistical properties of the Dirac operator and, in turn, influences the phase diagram.

The inclusion of quark chemical potentials, $\mu_{f}$, and the temperature, $T$, follows from the frequency representation of the partition function in the finite temperature formalism [6, 15]. The path integral is evaluated for fermion fields that obey antiperiodic boundary conditions in Euclidean time. Thus, the Fourier decomposition

$$
\psi(\tau)=T \sum_{\omega_{n}} e^{-\omega_{n} \tau} \psi\left(\omega_{n}\right)
$$

contains only the odd Matsubara frequencies

$$
\omega_{n}=i(2 n+1) \pi T \text {. }
$$

The dependence on $\mu_{f}$ and $T$ appears as a component of the Lagrangian of the form

$$
L_{1}=\int d^{4} x \sum_{f} \psi_{f}^{\dagger} \gamma_{4}\left(\partial_{\tau}+\mu_{f}\right) \psi_{f}
$$

whose Fourier representation is given as

$$
L_{1}=T \sum_{n} \int d^{3} \vec{x} \sum_{f} \psi_{f}^{\dagger}\left(\omega_{n}\right) \gamma_{4}\left(-i(2 n+1) \pi T+\mu_{f}\right) \psi_{f}\left(\omega_{n}\right)
$$


There is a certain freedom associated with the introduction of such a term in random matrix models. Equation (7) suggests that we should add a deterministic term with the block structure of $\gamma_{4}$ to the Dirac operator of Eq. (2). With Euclidean matrices, this gives

$$
D[W] \rightarrow D\left[W ; \mu_{f}, T\right]=\left(\begin{array}{cc}
0 & i W+\omega_{+}\left(\mu_{f}, T\right) \\
i W^{\dagger}+\omega_{-}\left(\mu_{f}, T\right) & 0
\end{array}\right)
$$

where $\omega_{ \pm}$are $n \times n$ matrices. Here, $n$ is a compound index which combines random matrix and Matsubara frequency indices. Because the ensemble of matrices $W$ is invariant under unitary $(\beta=2)$ or orthogonal $(\beta=1)$ basis transformations, many equivalent forms of $\omega_{ \pm}$can be adopted, and their detailed form cannot be specified uniquely. (We will also consider another transformation below.) We know, however, that the eigenvalues of $\omega_{ \pm}$must be those of the operator in Eq. (7). In the following, we restrict ourselves to the two lowest Matsubara frequencies, $\omega_{n}= \pm i \pi T$. The appropriate forms of $\omega_{ \pm}$must then have two series of $n / 2$-degenerate eigenvalues $\mu_{f} \pm i \pi T$.

We now turn to the various schemes proposed for implementing temperature dependence. In an early model of diquark condensation [8, 9] in QCD with two flavors, we considered random matrix interactions that included spin and color quantum numbers explicitly. This choice leads to an expanded block structure

$$
W=\sum_{\mu=0}^{3} \sum_{a=1}^{3}\left(\sigma_{\mu} \otimes \sigma_{a}\right) A_{\mu a}
$$

where $\sigma_{\mu}=(1, i \vec{\sigma})$ represents spin degrees of freedom with the Pauli matrices $\vec{\sigma}$, while $\sigma_{a}$ are the $S U(2)$ color matrices. The interactions are to be integrated over the real matrices $A_{\mu a}$ whose elements are drawn on a Gaussian distribution. The $W$ appearing in Eq. (9) is complex independent of the number of colors. Because the dependence on spin and color is deterministic, the model nevertheless has the appropriate number of degrees of freedom per matrix element. For example, for $N_{c}=2$ the Dirac operator $D[W]$ in Eq. (2) with the block structure of Eq. (9) satisfies the antiunitary symmetry of Eq. (3). More generally, we have shown that for $N_{c}=3$ and $N_{c}=2$ the eigenvalues of the Dirac operator $D[W]$ exhibit the spectral correlations expected for the chiral unitary and orthogonal ensembles, respectively 16$]$.

The deterministic part of the Dirac operator is given as

$$
\omega_{+}\left(\mu_{f}, T\right)=\omega_{-}\left(\mu_{f}, T\right)=\left(\mathbf{1}_{\text {spin }} \otimes \mathbf{1}_{\text {color }}\right)\left(\mu_{f}+i s_{f} \pi T\right)
$$

where the choice of $s_{f}= \pm 1$ follows a selection rule. First, $s_{f}$ is taken positive for flavor $f=1$ and negative for flavor $f=2$. The contribution from the opposite choice, $s_{1}=-1$ and $s_{2}=1$, is then added to obtain the thermodynamic potential. (In Refs. [8, 9], we considered the limit $\mu_{1}=\mu_{2}$; in that case, the two contributions are identical and the sum over pairs amounts to a factor of two.) This procedure is suggested by the Fourier expansion of the condensates in a microscopic theory. The chiral condensate is given by the equal time correlator $C_{\sigma} \sim\langle\bar{\psi}(\tau) \psi(\tau)\rangle$, which is also time independent. A frequency decomposition of the fields, Eq. (4), leads to an expression which contains a sum over all frequency pairs. Since the correlator $C_{\sigma}$ is time independent, the only non-vanishing terms are those for which the Fourier components $\bar{\psi}\left(\omega_{n}\right)$ and $\psi\left(\omega_{m}\right)$ have equal frequencies (or $\left.m=n\right): C_{\sigma} \sim T\left\langle\sum_{n} \bar{\psi}\left(\omega_{n}\right) \psi\left(\omega_{n}\right)\right\rangle$. Similarly, the diquark condensate is given by the equal-time correlator $C_{\Delta} \sim\left\langle\psi^{T}(\tau)\left(i \sigma_{2}\right)_{\text {color }}\left(i \sigma_{2}\right)_{\text {spin }}\left(i \sigma_{2}\right)_{\text {flavor }} \psi(\tau)\right\rangle$ and is constant in time $\tau$. A Fourier decomposition now selects opposite frequencies for the two fields, which necessarily correspond to different flavors because of $\left(i \sigma_{2}\right)_{\text {flavor }}$. This mechanism was implemented in the random matrix model through the selection of appropriate pairs $\left(s_{1}, s_{2}\right)$.

In recent work 10, 11], Klein, Toublan, and Verbaarschot treated the chiral random matrix ensembles, studying diquark condensation for the case $N_{c}=2$ only. The zero temperature and zero $\mu_{f}$ version of their model follows Eq. (1) with $W$ complex for the unitary ensemble and real for the orthogonal ensemble. In their approach, the dependence on $\mu_{f}$ and $T$ follows the symmetries of the chiral ensembles. For the unitary ensembles, $\omega_{ \pm}$are complex. In the appropriate sub-basis of frequency states, they assume the diagonal form

$$
\omega_{+}\left(\mu_{f}, T\right)=\omega_{-}\left(\mu_{f}, T\right)=\left(\begin{array}{cc}
\mu_{f}+i \pi T & 0 \\
0 & \mu_{f}-i \pi T
\end{array}\right),
$$

where each block matrix has dimension $n / 2 \times n / 2$. For the chiral orthogonal ensembles, the antiunitary symmetry of Eq. (3) is imposed, which leads to a particular basis for which the $\omega_{ \pm}$matrices are real. Ensuring that their eigenvalues are given as $\mu_{f} \pm i \pi T$, the proposed dependence is

$$
\begin{aligned}
& \omega_{+}=\left(\begin{array}{cc}
\mu_{f} & \pi T \\
-\pi T & \mu_{f}
\end{array}\right), \\
& \omega_{-}=\omega_{+}^{T},
\end{aligned}
$$


where the dimensions of the block matrices are again $n / 2 \times n / 2$.

At first sight, the two forms of $\omega_{ \pm}$, Eqs. (11) and (12), might seem non-equivalent and also different from that of Eq. (10). There thus appears to be a disagreement between the temperature dependence that is obtained from a generic structure suggested by symmetry and one which is based on physical knowledge of the frequency couplings that are allowed. In the next section, we consider the case $N_{c}=2$ and show that the dependence expressed by the selection rule in Eq. (10) leads to the same effective thermodynamic potential as that obtained with the dependence of Eq. (12). We comment on the correct form to be used for $N_{c}=3$ in Sec. III]

\section{EQUIVALENCES BETWEEN THE TEMPERATURE SCHEMES}

It is useful to compare the temperature schemes of Eqs. (10), (11), and (12) in a common framework. To this end, we recast the model developed in [8, 9] in a form close to that of Refs. 10, 11]. We consider a model for $N_{c}=2$, two flavors, and zero quark masses. We work with an interaction $W$ having the expanded block-structure of Eq. (9) and derive the thermodynamic potential for the partition function of Eq. (1). After integrating over the random matrices $A_{\mu a}$ and performing a Fierz transformation, we obtain the partition function [9]

$$
Z=\prod_{f} \int \mathcal{D} \psi_{f} \mathcal{D} \psi_{f}^{\dagger} \exp (Y) \exp \left(\sum_{f=1,2} \psi_{f}^{\dagger}\left(\begin{array}{cc}
0 & \omega_{+}\left(\mu_{f}, T\right) \\
\omega_{-}\left(\mu_{f}, T\right) & 0
\end{array}\right) \psi_{f}\right),
$$

where $Y$ represents the four-point interaction. If only the chiral and diquark channels are retained,

$$
\begin{aligned}
Y= & \frac{3}{4 n \beta \Sigma^{2}}\left(\left(\psi_{R 1}^{\dagger} \cdot \psi_{R 1}\right)\left(\psi_{L 1}^{\dagger} \cdot \psi_{L 1}\right)+\left(\psi_{R 2}^{\dagger} \cdot \psi_{R 2}\right)\left(\psi_{L 2}^{\dagger} \cdot \psi_{L 2}\right)+\right. \\
& \left.\left(\psi_{R 1}^{\dagger} \cdot P_{\Delta} \cdot \psi_{R 2}^{*}\right)\left(\psi_{L 2}^{T} \cdot P_{\Delta} \cdot \psi_{L 1}\right)+\left(\psi_{L 1}^{\dagger} \cdot P_{\Delta} \cdot \psi_{L 2}^{*}\right)\left(\psi_{R 2}^{T} \cdot P_{\Delta} \cdot \psi_{R 1}\right)\right) .
\end{aligned}
$$

Here, we have adopted the notation $\phi \cdot \chi \equiv \sum_{i=1}^{n} \phi_{i} \chi_{i}$, where $i$ is the random matrix index, and have introduced the projector $P_{\Delta}=\left(i \sigma_{2}\right)_{\text {spin }}\left(i \sigma_{2}\right)_{\text {color }}$.

Following [6] and [1], bosonic auxiliary variables are introduced via a Hubbard-Stratonovich transformation that accounts for the different combinations of spin, color, and flavor quantum numbers. This transformation is given by

$$
e^{Y}=\int \mathcal{D} A e^{-\frac{n \beta \Sigma^{2}}{6} \operatorname{Tr} A A^{\dagger}} e^{\mathcal{Y}(A)}
$$

where $A$ is a complex, antisymmetric $16 \times 16$ matrix, and $\mathcal{Y}(A)$ is the bilinear

$$
\mathcal{Y}(A)=\sum_{f=1,2} \frac{1}{2}\left(\begin{array}{c}
\psi_{R}^{f} \\
\psi_{R}^{f *}
\end{array}\right)^{T}\left(A^{\dagger} \otimes \mathbf{1}_{n}\right)\left(\begin{array}{c}
\psi_{R}^{f} \\
\psi_{R}^{f *}
\end{array}\right)+\sum_{f=1,2} \frac{1}{2}\left(\begin{array}{c}
\psi_{L}^{f} \\
\psi_{L}^{f *}
\end{array}\right)^{T}\left(B \otimes \mathbf{1}_{n}\right)\left(\begin{array}{c}
\psi_{L}^{f} \\
\psi_{L}^{f *}
\end{array}\right) .
$$

Here, $\mathbf{1}_{n}$ represents the identity matrix in the combined space of random matrix/frequency indices and $B$ is given as

$$
B=\left(\begin{array}{ll}
0 & 1 \\
1 & 0
\end{array}\right) A\left(\begin{array}{ll}
0 & 1 \\
1 & 0
\end{array}\right)
$$

In a basis of states $\left(\psi_{f}, \psi_{f}^{*}\right)=\left(\psi_{1}, \psi_{2}, \psi_{1}^{*}, \psi_{2}^{*}\right)$, the matrix $A$ assumes the form

$$
A=\left(\begin{array}{cccc}
0 & \Delta_{R} P_{\Delta} & \sigma_{1}^{*} & 0 \\
-\Delta_{R} P_{\Delta} & 0 & 0 & \sigma_{2}^{*} \\
-\sigma_{1}^{*} & 0 & 0 & -\Delta_{L}^{*} P_{\Delta} \\
0 & -\sigma_{2}^{*} & \Delta_{L}^{*} P_{\Delta} & 0
\end{array}\right)
$$

where each block is $4 \times 4$ (two spins $\times$ two colors), and the auxiliary variables $\Delta_{L}, \Delta_{R}, \sigma_{1}$, and $\sigma_{2}$ are complex scalars.

The temperature and chemical potential terms of Eq. (13) can be arranged as

$$
\begin{aligned}
\sum_{f=1,2} \psi_{f}^{\dagger}\left(\begin{array}{cc}
0 & \omega_{+} \\
\omega_{-} & 0
\end{array}\right) \psi_{f}= & \sum_{f=1,2} \frac{1}{2}\left(\begin{array}{c}
\psi_{R}^{f} \\
\psi_{R}^{f *}
\end{array}\right)^{T}\left(\begin{array}{cc}
0 & -\omega_{+}^{T} \\
\omega_{+} & 0
\end{array}\right)\left(\begin{array}{c}
\psi_{L}^{f} \\
\psi_{L}^{f *}
\end{array}\right) \\
& +\sum_{f=1,2} \frac{1}{2}\left(\begin{array}{c}
\psi_{L}^{f} \\
\psi_{L}^{f *}
\end{array}\right)^{T}\left(\begin{array}{cc}
0 & -\omega_{-}^{T} \\
\omega_{-} & 0
\end{array}\right)\left(\begin{array}{c}
\psi_{R}^{f} \\
\psi_{R}^{f *}
\end{array}\right) .
\end{aligned}
$$


Combining Eqs. (13), (15), and (19) and integrating over the fermion fields, we finally obtain

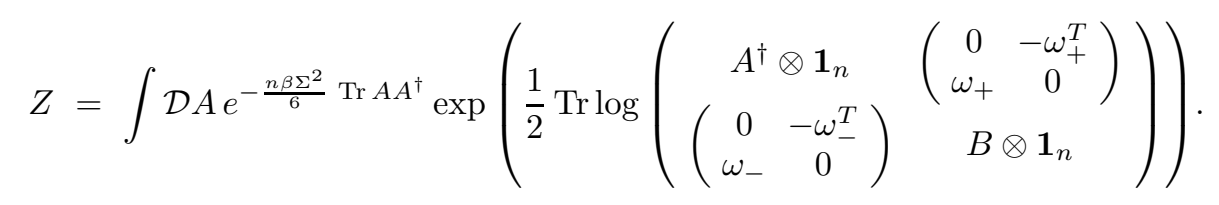

For later discussions, it is important to note that the diagonal blocks of the argument of the logarithm are given as an exterior product with $\mathbf{1}_{n}$. Different Matsubara frequencies will thus correspond to the same auxiliary matrix, $A^{\dagger}$ or $B$.

Equation (20) is the central expression which we will use to compare the different temperature schemes. We will concentrate on the same condensation channels as those considered earlier [8, 9]. We take equal quark chemical potentials, $\mu_{1}=\mu_{2}=\mu$. The chiral condensate is considered to be flavor independent so that $\sigma_{1}=\sigma_{2}=\sigma$. Moreover, we have $\left\langle\psi_{2 R}^{T} P_{\Delta} \psi_{1 R}\right\rangle=\left\langle\psi_{2 L}^{T} P_{\Delta} \psi_{1 L}\right\rangle$, and the auxiliary fields satisfy the equality $\Delta_{R}=\Delta_{L}=\Delta_{\text {. }}$.

\section{A. Selection rule scheme}

The temperature dependence proposed in Refs. [8, 9] can now be represented as $\left(\mu_{1}=\mu_{2}=\mu\right)$

$$
\left\{\begin{aligned}
\omega_{+}\left(\mu_{1}, T\right)=\omega_{-}\left(\mu_{1}, T\right) & =\left(\begin{array}{cc}
\mu+i \pi T & 0 \\
0 & \mu-i \pi T
\end{array}\right)_{\text {Matsubara }} \\
\omega_{+}\left(\mu_{2}, T\right)=\omega_{-}\left(\mu_{2}, T\right) & =\left(\begin{array}{cc}
\mu-i \pi T & 0 \\
0 & \mu+i \pi T
\end{array}\right)_{\text {Matsubara }}
\end{aligned}\right.
$$

Inserting this form in Eq. (20) leaves us with a straightforward evaluation of a trace. We obtain

$$
Z=\int \mathcal{D} A e^{-4 n \Omega}
$$

with an effective thermodynamic potential

$$
\Omega(\sigma, \Delta)=\frac{2 \beta \Sigma^{2}}{3}\left(\sigma^{2}+|\Delta|^{2}\right)-\log \left((\sigma-\mu)^{2}+|\Delta|^{2}+\pi^{2} T^{2}\right)-\log \left((\sigma+\mu)^{2}+|\Delta|^{2}+\pi^{2} T^{2}\right)
$$

(The inclusion of a quark mass $m_{f}=m$ would amount to replace $\sigma$ by $\sigma+m$ in the argument of the logarithm.) In the thermodynamic limit $n \rightarrow \infty$, one can evaluate the partition function integral exactly by a saddle point method and obtain

$$
\lim _{n \rightarrow \infty} Z \sim e^{-4 n \Omega\left(\sigma_{\text {saddle }}, \Delta_{\text {saddle }}\right)}
$$

where the fields $\sigma_{\text {saddle }}$ and $\Delta_{\text {saddle }}$ are solutions of the gap equations

$$
\begin{aligned}
& \frac{\partial \Omega}{\partial \sigma}(\sigma, \Delta)=0, \\
& \frac{\partial \Omega}{\partial \Delta}(\sigma, \Delta)=0 .
\end{aligned}
$$

Although the potential in Eq. [23) has been obtained using a formalism different from that used in Ref. 8], we recover the earlier result. We now turn to other temperature schemes.

\section{B. A selection rule scheme with explicit flavor symmetry}

At first sight, the temperature dependence in Eq. (21) might seem to violate flavor symmetry. However, this is not the case. The sign reversal in $T$ for flavor 2 can also be expressed as a permutation of the two Matsubara frequencies, since

$$
-\left(\begin{array}{cc}
i \pi T & 0 \\
0 & -i \pi T
\end{array}\right)=\left(\begin{array}{ll}
0 & 1 \\
1 & 0
\end{array}\right)\left(\begin{array}{cc}
i \pi T & 0 \\
0 & -i \pi T
\end{array}\right)\left(\begin{array}{ll}
0 & 1 \\
1 & 0
\end{array}\right)
$$


Therefore, we can go to an equivalent representation by permuting the frequency states for flavor 2 . The selection of opposite frequencies applies to diquark and antidiquark bilinears for which the projector $P_{\Delta}$ is sandwiched between the flavor fields as in Eq. (14). Hence, the effect of permuting positive and negative frequencies for flavor 2 transforms $P_{\Delta}$ into

$$
P_{\Delta} \rightarrow \tilde{P}_{\Delta}=P_{\Delta}\left(\begin{array}{ll}
0 & 1 \\
1 & 0
\end{array}\right)_{\text {Matsubara }}=\left(i \sigma_{2}\right)_{\text {spin }} \otimes\left(i \sigma_{2}\right)_{\text {color }} \otimes\left(\begin{array}{ll}
0 & 1 \\
1 & 0
\end{array}\right)_{\text {Matsubara }} .
$$

By contrast, the chiral bilinears are unaffected by the frequency permutation, e.g., $\psi^{2 \dagger} \psi^{2}=\psi_{+}^{2 \dagger} \psi_{+}^{2}+\psi_{-}^{2 \dagger} \psi_{-}^{2}$. The sole effect of permuting the frequencies for flavor 2 is thus to make the frequency selection mechanism appear in the structure of the order parameter through the projector $\tilde{P}_{\Delta}$. The frequency component of this operator is flavor diagonal and $\tilde{P}_{\Delta}$ therefore respects the required flavor symmetries.

\section{Antisymmetric temperature dependence}

The apparent disagreement between a temperature dependence based on symmetry and one based on selecting frequency couplings would immediately be resolved by a transformation relating Eqs. (12) and (10). In the antisymmetric representation of Eq. (12), the basis of states is meant to be that for which all matrix elements of $W$ are real. This is certainly not the basis set to be used in our model, Eq. (13), since the spin and color dependence of Eq. (9) makes $W$ complex. Nevertheless, the Dirac operator $D[W]$ of Eq. (2) satisfies the anti-unitary symmetry of Eq. (3), and we could in principle choose the appropriate spin and color basis to obtain a real $W[\underline{8}$. This is a consequence of the fact that the matrix elements contain the proper number of random degrees of freedom. In the following we are interested in the transformation properties of the deterministic temperature terms $\omega_{ \pm}$and thus do not consider transformations in spin/color space. We focus instead on the effects of a partial transformation acting only on frequency indices.

We wish to establish the relationship between the temperature dependence based on the selection rule of Eq. (28) and an antisymmetric dependence. We start from the representation of Sec. IIB for which

$$
\omega_{ \pm}=\mu+\left(\begin{array}{cc}
i \pi T & 0 \\
0 & -i \pi T
\end{array}\right)
$$

and the diquark order parameter contains the projector $\tilde{P}_{\Delta}$ of Eq. (28). We rewrite the diagonal temperature form as

$$
\left(\begin{array}{cc}
i \pi T & 0 \\
0 & -i \pi T
\end{array}\right)=U_{m}\left(\begin{array}{cc}
0 & \pi T \\
-\pi T & 0
\end{array}\right) U_{m}^{\dagger}
$$

where

$$
U_{m}=\frac{1}{\sqrt{2}}\left(\begin{array}{cc}
1 & -i \\
1 & i
\end{array}\right)
$$

is unitary. We then perform the transformation

$$
\begin{aligned}
& \psi_{R, L}=U \psi_{R, L}^{\prime}, \\
& \psi_{R, L}^{*}=U^{*} \psi_{R, L}^{\prime *},
\end{aligned}
$$

with

$$
U=\left(\begin{array}{cc}
U_{m} \otimes \mathbf{1}_{n / 2} & 0 \\
0 & U_{m} \otimes \mathbf{1}_{n / 2}
\end{array}\right) \otimes \mathbf{1}_{\text {spin }} \otimes \mathbf{1}_{\text {color }} \otimes \mathbf{1}_{\text {flavor }} .
$$

This transformation leaves the partition function of Eq. (20) unaffected. Since $U$ only acts in frequency space, it does not affect the quadratic term, $\operatorname{Tr} A A^{\dagger}$. The logarithmic term is also unaltered since $\operatorname{Tr} \log M=\log \operatorname{det} M$, and the determinant is invariant under the transformation

$$
M \rightarrow M^{\prime}=\left(\begin{array}{cc}
U^{T} & 0 \\
0 & U^{\dagger}
\end{array}\right) M\left(\begin{array}{cc}
U & 0 \\
0 & U^{*}
\end{array}\right)
$$

Hence, the transformation, $U$, brings the temperature representation of Sec. IB to an equivalent representation with an antisymmetric temperature dependence. 
We now examine the transformation of the bilinear form of Eq. 16) bearing in mind that we started with the representation of Sec. [IB for which the diquark terms contain the projector $\tilde{P}_{\Delta}$ of Eq. (28). Mesonic bilinears of the form $\psi_{i}^{\dagger} \psi_{j}$ (where $i$ and $j$ represent some combination of spin/color/flavor indices) are invariant under the $U$-transformation as

$$
\psi_{i}^{\dagger} \psi_{j}=\psi_{i}^{\dagger^{\prime}} U^{\dagger} U \psi_{j}^{\prime}=\psi_{i}^{\dagger^{\prime}} \psi_{j}^{\prime}
$$

By contrast, diquark bilinears of the form $\psi_{i}^{T} \tilde{P}_{\Delta} \psi_{j}$ are transformed to

$$
\psi_{i}^{T} \tilde{P}_{\Delta} \psi_{j}=\psi_{i}^{\prime T} P_{\Delta}^{\prime} \psi_{j}^{\prime},
$$

with

$$
P_{\Delta}^{\prime}=U_{m}^{T} \tilde{P}_{\Delta} U_{m}=P_{\Delta},
$$

where again, $P_{\Delta}=\left(i \sigma_{2}\right)_{\text {spin }}\left(i \sigma_{2}\right)_{\text {color }}$ is block diagonal in frequency space.

To summarize, the temperature dependence in the new basis is antisymmetric, while the diquark order parameter no longer contains an explicit frequency selection operator. This is precisely the form proposed in Ref. [11]. We thus have shown that it is equivalent to the selection rule of Eq. (28) and, by extension, to the one originally proposed in Ref. [8, 9]. To complete the proof, it is readily verified that the various temperature schemes of Eqs. (10), (12), and (28), used with the corresponding forms for the diquark projector $\left(P_{\Delta}, \tilde{P}_{\Delta}\right.$, or $\left.P_{\Delta}^{\prime}=P_{\Delta}\right)$, all yield the thermodynamic potential of Eq. (23).

\section{Pion condensates}

Although the temperature dependence in Eq. (10) produces the correct result for modelling diquark and chiral condensation, it is inconvenient if one also wishes to consider the possibility of forming a pion condensate, $\left\langle\psi^{\dagger} \gamma_{5}\left(i \sigma_{2}\right) \psi\right\rangle$. Pion bilinears must be taken between fields of equal frequencies and opposite flavors, whereas elements associated with diquark bilinears require opposite frequencies and opposite flavors. Considering both channels thus leads to some tedious bookkeeping. However, the equivalent form of Eq. (28) can be implemented much more easily and extended to the inclusion of pion bilinears. The resulting form takes a diagonal temperature dependence, considers diquark bilinears with an off-diagonal projector $\tilde{P}_{\Delta}$, while it leaves both the chiral and pion bilinears diagonal in frequency space. We now show that such prescription gives the same results as those obtained with an antisymmetric temperature dependence.

A Fierz projection of the random matrix interactions on the pion condensation channel yields a four fermion component

$$
Y_{\pi}=\frac{3}{32 n \beta \Sigma^{2}}\left(\psi^{\dagger} \gamma_{5}\left(i \sigma_{2}\right)_{\text {flavor }} \psi\right)^{2}
$$

Using again a Hubbard-Stratonovich transformation to introduce an associated auxiliary variable, $\rho$, one is led to consider the auxiliary matrix

$$
A=\left(\begin{array}{cccc}
0 & \Delta_{R} P_{\Delta} & \sigma_{1}^{*} & \rho \\
-\Delta_{R} P_{\Delta} & 0 & -\rho & \sigma_{2}^{*} \\
-\sigma_{1}^{*} & \rho & 0 & -\Delta_{L}^{*} P_{\Delta} \\
-\rho & -\sigma_{2}^{*} & \Delta_{L}^{*} P_{\Delta} & 0
\end{array}\right)
$$

As previously, the frequency selection rule is introduced by replacing $P_{\Delta}$ by $\tilde{P}_{\Delta}$ in the diquark and antidiquark bilinears of Eq. (16) or equivalently in the argument of the logarithm of Eq. (20). Such a temperature scheme is completely consistent with the spirit with which we derived the temperature dependence in Refs. [8, [9]; we have verified that it produces the same thermodynamical potential as that obtained with an antisymmetric temperature dependence. The result is identical to that of Ref. [11].

\section{DISCUSSION AND CONCLUSIONS}

For $N_{c}=2$, we have resolved the apparent disagreement between the dependence based on an antisymmetric temperature term and that based on a frequency selection rule. The two representations are related by a unitary 
transformation which leaves the thermodynamic potential unchanged and which therefore does not affect physical observables. The change of basis is, however, reflected in the structure of the diquark order parameter or, equivalently, in the expression for the frequency selection rule.

Technically, temperature enters through the imposition of an antiperiodic boundary condition on the fermion fields. There is a certain degree of freedom in representing temperature terms in a random matrix model. However, as soon as one imposes a given block structure (e.g. diagonal or antisymmetric), one is actually selecting a specific basis for the frequency states. As a result, attention must be paid to the physical meaning of the basis states when solving for the partition function and, accordingly, in the structure of the order parameter. If a change of basis appears useful for mathematical convenience, one must naturally keep track of the effects of such transformation on the states and transform the order parameter accordingly. From a general point of view, any transformation with the block diagonal form of Eq. (34) with $U_{m}$ unitary leaves the thermodynamic potential invariant. There are thus many equivalent Matsubara bases which can be chosen and correspondingly many correct associated forms of $\omega_{ \pm}$and of the diquark order parameter. Among these forms, the antisymmetric one appears to be particularly convenient since the corresponding diquark projector is block diagonal in frequency space. The price to be paid for working with a different form is merely the need to rely on the knowledge of the physical meaning of the basis states and on which frequency couplings are allowed. This will determine the form of the corresponding diquark projector.

The situation is not very different for QCD with $N_{c}=3$. If one chooses a diagonal temperature term, one still needs to select opposite frequencies for the fields involved in the diquark condensate. In this case, however, the frequency selection mechanism cannot be revealed by symmetry arguments alone since QCD with $N_{c}=3$ does not satisfy an antiunitary symmetry. As above, there are many correct forms of $\omega_{ \pm}$which can be used together with the corresponding prescriptions for the frequency couplings. In Ref. [10], the authors used the diagonal form of Eq. (11) but did not consider diquark condensates. Thus, there was no need for specifying a diquark projector, and the choice of a diagonal form of $\omega_{ \pm}$was as correct as any other. On the other hand, diquark condensation was investigated in Ref. [8, [9] using a diagonal form. There, it was necessary to supplement the temperature dependence with an explicit frequency selection rule.

In conclusion, the proper temperature dependence to be used in a given random matrix model depends primarily on the form of the condensates that may develop. Symmetry arguments are useful in identifying the mechanism for selecting appropriate frequencies, but they are not always sufficient.

\section{Acknowledgments}

J. J. M. Verbaarschot is acknowledged for a valuable discussion.

[1] B. Barrois, Nucl. Phys. B129 (1977) 390; D. Bailin and A. Love, Phys. Rept. 107 (1984) 325.

[2] M. Alford, K. Rajagopal, and F. Wilczek, Phys. Lett. B 422 (1998) 247; R. Rapp, T. Schäfer, E. V. Shuryak, and M. Velkovsky, Phys. Rev. Lett. 81 (1998) 53.

[3] K. Rajagopal and F. Wilczek, in B. L. Ioffe Festschrift, At the Frontier of Particle Physics/Handbook of QCD, M. Shifman ed., (World Scientific 2001); M. Alford, Ann. Rev. Nucl. Part. Sci. 51 (2001) 131; T. Schäfer, hep-ph/0304281 H.-c. Ren, hep-ph/0404074

[4] M. A. Halasz, A. D. Jackson, R. E. Shrock, M. A. Stephanov, and J. J. M. Verbaarschot, Phys. Rev. D 58 (1998) 096007.

[5] K. Iida and G. Baym, Phys. Rev. D 63 (2001) 074018; Phys. Rev. D 66 (2002) 059903(E); Phys. Rev. D 66 (2002) 014015; I. Giannakis and H.-C. Ren, Nucl.Phys. B669 (2003) 462.

[6] A. D. Jackson and J. J. M. Verbaarschot, Phys. Rev. D 53 (1996) 7223.

[7] R. A. Janik, M. A. Nowak, G. Papp, and I. Zahed, Nucl. Phys. A642 (1998) 191c.

[8] B. Vanderheyden and A. D. Jackson, Phys. Rev. D 64 (2001) 074016.

[9] B. Vanderheyden and A. D. Jackson, Phys. Rev. D 61 (2000) 076004; Phys. Rev. D 62 (2000) 094010.

[10] B. Klein, D. Toublan, and J. J. M. Verbaarschot, Phys. Rev. D 68 (2003) 014009.

[11] B. Klein, D. Toublan, and J. J. M. Verbaarschot, arXiv:hep-ph/0405180.

[12] E. Shuryak and J. Verbaarschot, Nucl. Phys. A560 (1993) 306.

[13] J. Verbaarschot and I. Zahed, Phys. Rev. Lett. 70 (1993) 3852.

[14] J. J. M. Verbaarschot, Phys. Rev. Lett. 72 (1994) 2531.

[15] For the finite temperature formalism in path integral representations, see for instance Quantum many-particle systems, J. W. Negele and H. Orland, (Westview Press, Boulder, 1988).

[16] B. Vanderheyden and A. D. Jackson, Phys. Rev. D 67 (2003) 085016. 